\title{
Microstructural Aspects of Wedge, Craze, and Brittle Modes of Deformation in Thermoplastic Materials
}

\author{
A. Dasari and R.D.K. Misra \\ Materials Science and Engineering Group, Department of Chemical Engineering, University of \\ Louisiana at Lafayette, P.O. Box 44130, Lafayette, LA 70504-4130, USA (dmisra@louisiana.edu).
}

The mode of fracture in materials reflects the sensitivity of a material to the applied stress. Scanning electron microscopy (SEM) and atomic force microscopy (AFM) are relevant techniques that provide an understanding of mechanisms of deformation and fracture in materials. Although, both AFM and SEM have similar lateral resolution, AFM has superior vertical resolution and provides three dimensional, quantitative information of the surface topography and the morphology of the localized deformation processes at the micro- to pico-scale levels [1]. In the present study, the potential of AFM and SEM to understand tensile deformation processes such as wedging, crazing-tearing, and brittle mode of failure (Figure 1) in thermoplastic materials is presented.

Wedging is a common surface deformation process in high density polyethylene (HDPE) and ethylene-propylene copolymers. During the early stages of tensile straining, wedges are initially nucleated on the deformed surface in the direction of tensile stress. With increase in \% strain, the wedges multiply and coalesce with previously formed wedges. On continued straining, the wedges are separated from the matrix as long and fine deformation fibrils with large number of voids leading to fibrillated type of fracture (defined as highly ductile drawing of fibrils with enhanced plastic flow) [1]. AFM studies of the surface morphology of the wedge-containing region suggest that during tensile straining, fibrils/microfibrils are preferentially aligned in the tensile direction (Figure 2). However, in comparison with the undeformed surface morphology, the fibrils in the wedge region are closely packed, oriented, and excessively stretched along the tensile direction, leading to reduction in surface height. This excessive stretching of fibrils confirms that the wedge deformation process is associated with enhanced microplasticity and is representative of ductile failure.

In polypropylene (PP), during the early stages of deformation, surface features referred as 'deformation bands' are formed normal to the tensile stress direction. The deformation bands are regions of highly localized deformation. When a crack propagates through them, craze-like features develop perpendicular to the tensile loading direction. These crazes grow inwards and the final fracture occurs by a combination of craze and brittle mode of fracture; the percentage of which depends on the tensile loading rate. In general, a craze residue in a material is described as a microscopic region of numerous tiny surface cracks, consisting of an interpenetrating system of highly oriented molecules separated by voids. AFM of the craze residue (Figure 3) indicated that the fibrils/microfibrils are oriented and aligned in the direction of tensile axis, and are characterized by splitting (or breakage) of fibrils normal to the alignment direction that leads to void formation between the drawn fibrils. The process of orientation and stretching of fibrils along the tensile direction within the craze is associated with merging of fibrils. The merged fibrils breakdown resulting in bands of voids, and finally a microcrack (craze) bridged by fibrils are formed.

AFM of the brittle region (Figure 4) suggested that brittle mode of deformation involves partial reorganization of microstructure, involving instantaneous stretching process followed by a pull-back 
of the microstructure in the direction opposite to the tensile force. Thus, SEM in association with AFM provides an insight of the micromechanisms of deformation.

\section{References}

[1]. A. Dasari, J. Rohrmann, and R.D.K. Misra, Macromol. Mater. and Engg., 287, (2002) 889.
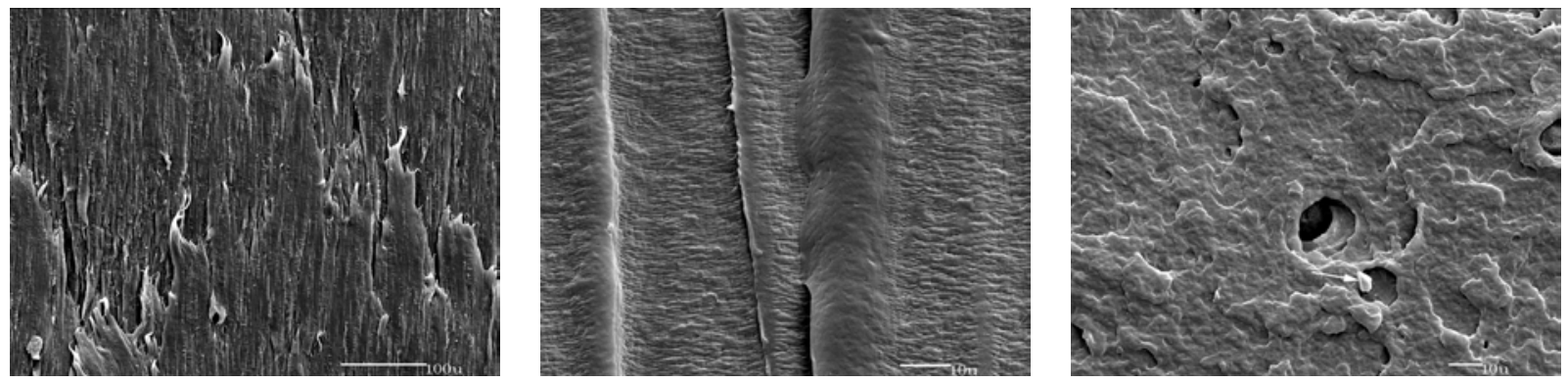

Figure 1. Micromechanisms of deformation, (a) wedge, (b) crazing/tearing, and (c) brittle.
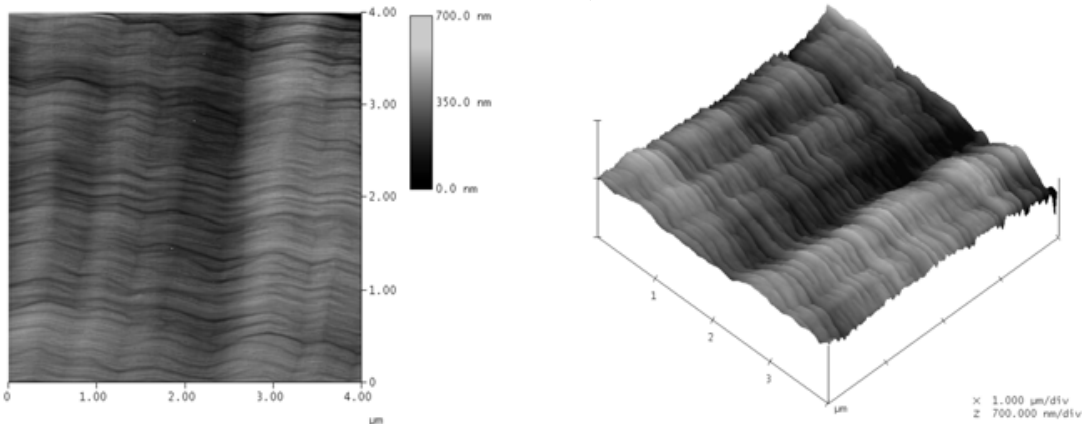

Figure 2. 2-D and 3-D AFM micrographs of wedge residue in HDPE.
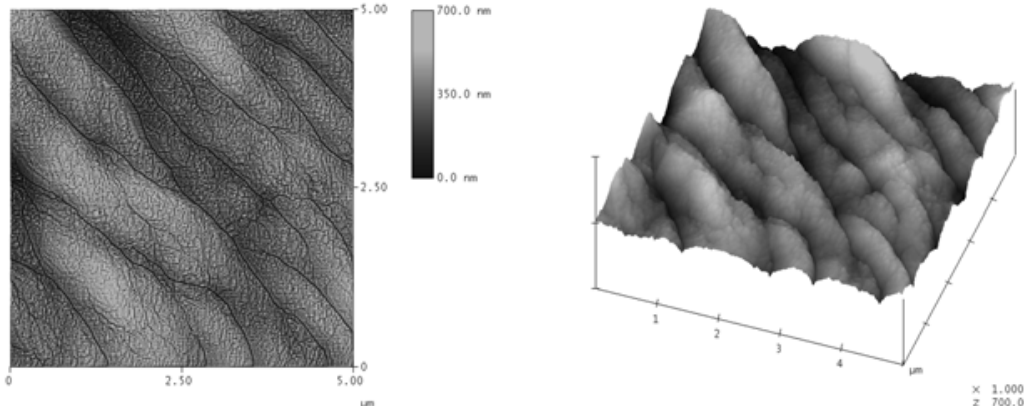

Figure 3. 2-D and 3-D AFM micrographs of craze residue in PP.

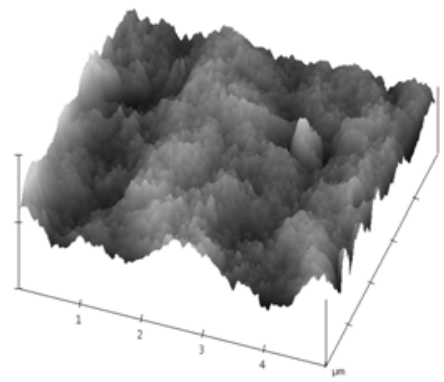

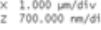

Figure 4. 3-D AFM micrograph of brittle mode of deformation in PP. 